\title{
UkrVO Astroinformatics Software and Web-services
}

\author{
I. B. Vavilova ${ }^{1}$, Ya. S. Yatskiv ${ }^{1}$, L. K. Pakuliak ${ }^{1}$, I. L. Andronov ${ }^{2}$, \\ V. M. Andruk ${ }^{1}$, Yu. I. Protsyuk ${ }^{3}$, V. E. Savanevych ${ }^{4}$, \\ D. O. Savchenko ${ }^{5}$ and V. S. Savchenko ${ }^{6}$ \\ ${ }^{1}$ Main Astronomical Observatory, National Academy of Sciences of Ukraine, \\ 27 Akademika Zabolotnogo St., Kyiv 03143, Ukraine \\ ${ }^{2}$ Odesa National Maritime University, 34 Mechnikova St., Odesa 65029, Ukraine \\ ${ }^{3}$ Nikolaev Astronomical Observatory, 1 Observatorna St., Mykolaiv 54030, Ukraine \\ ${ }^{4}$ Western Radio Technical Surveillance Center, \\ National Space Agency of Ukraine, Kosmonavtiv St., Mukachevo 89600, Ukraine, \\ ${ }^{5}$ Bogolyubov Institute of Theoretical Physics, National Academy of Sciences of Ukraine, \\ 14b, Metrologichna St., Kyiv 03143, Ukraine \\ ${ }^{6}$ Institut national de physique nuclaire et de physique des particules, CNRS \\ 3 rue Michel-Ange, 75794, Paris Cedex 16, France
}

\begin{abstract}
The aim of this paper is to introduce the software and astronomical web-services developed in frame of the Ukraine Virtual Observatory (UkrVO) and VIrtual Roentgen and Gamma Observatory (VIRGO) to the broad astronomical community. We report briefly on such web-services as the public databases "Sky Maps" of X-ray observations by XMM-Newton, the Earth Orbital Parameters for International Earth Rotation System, and the UkrVO Joint Digitized Archive of astroplates. We refer to such software as the "Multi-Column View", "Variable Stars Calculator", "FrameSmooth" for variable star's research and "Collection Light Technology" (CoLiTec) software for search and discovery of new Solar System bodies.
\end{abstract}

Keywords. astronomical data bases: miscellaneous

\section{Introduction}

For centuries the collection and distribution of the astronomical data have played a key role for conducting a scientific research. In the 21st century this activity has been resulting in the formation of the Astroinformatics as a new reserch field. "The experiences with data are now increasingly more virtual (through online databases) than physical (through trips to mountaintop observatories)" (cited by Borne et al. 2009). Astroinformatics provides a new data-oriented approach and advanced methodology for processing astronomical survey and catalogues with their teta- to peta-scale astroinformation resource over entire electromagnetic spectrum (see, for example, Eastman et al. 2005, Gray et al. 2005, Butler 2007, Cavuoti 2012, Vavilova et al. 2012a, Goodman et al. 2012, Brescia \& Longo 2013, Norris et al. 2013, Ruiz et al. 2014).

The crucial role in promulgating new methods and services to the astronomy community belongs to the International Virtual Observatory Alliance (IVOA). At the beginning of the era of big data the IVOA has initiated a creation of the national virtual observatories and development of the infrastructure of astronomical data center. Such centers give to users handy tools for data selection from large astronomical catalogues for a relatively small region of interest on the sky, data standards and services for image processing (likely to Aladin), manuals for processing the raw space-born data of various space missions (Chandra, XMM-Newton, Swift, NuStar, WISE, Kepler, GAIA, etc.). Besides the 
virtual observatories there are other sources for public outreach, for example, the Dataverse Network (Pepe et al. 2014), the Astrostatistics and Astroinformatics Portal (ASAIP, see, Feigelson et al. 2013, data centers and systems as the NED, HEASARC, SkyHound, NStED, SIMBAD, Planetary DS, and others (for a brief review on the multiwavelength surveys, see, for example, Michaelian 2016).

Ukraine Virtual Observatory (UkrVO) has been the IVOA member since 2011 (http://ukr-vo.org/). The current UkrVO supports the development of the 1) spectral and positional databases of celestial objects for multiwavelength studies of their properties, 2) software, and 3) digitized astro photo plate archives in the form of direct images of certain sky areas and celestial objects (Vavilova et al. 2012b, Vavilova 2016). VIrtual Roentgen and Gamma Observatory (VIRGO) for cosmology and high-energy astrophysics (http://virgoua.org/) is also a segment of VO "Infrastructure", which deals with ensuring the provision of standards for Grid Services of virtual organizations.

The aim of this paper is to introduce astroinformatics software and web-services developed in frame of the UkrVO and VIRGO to the broader astronomical community.

\section{UkrVO Software}

The powerful CoLiTec (Collection Light Technology) software for the automatic detection of small celestial objects (asteroids, for instance) on a series of CCD-frames has been developed by V. Savanyvych with collaborators (http://www.neoastrosoft.com). The core of this software is a new iteration method based on a subpixel Gaussian model of the discrete object image to estimate coordinates of celestial body. The method operates by continuous parameters (object's coordinates) in a discrete observational space (the set of pixel potentials) of the CCD-frame. In this model, the kind of coordinate distribution of the photons hitting a pixel of the CCD-frame is known a priori, while the associated parameters are determined from a real digital object image. The method is flexible in adapting to any form of object image, it has a high measurement accuracy along with a low calculating complexity (Savanevych et al. 2015a).

The comparative analysis of the processing of the same frames using CoLiTec and Astrometrica software says that in the case of low signal to noise ratios, the standard deviation of positional CCD-measurements using the Astrometrica software is 30-50\% greater than that of the CoLiTec software (Savanevych et al. 2015b). Since 2010, CoLiTec software has been installed at several observatories of the world with the aim of automatic discoveries of asteroids and comets on a set of CCD-frames. As the result, four comets C/2010 X1 (Elenin), P/2011 NO1 (Elenin), C/2012 S1 (ISON), and P/2013 V3 (Nevski) as well as more than 1500 small Solar System bodies were discovered. In 2014, this software was recommended to all members of the Gaia-FUN-SSO network for analyzing observations as a tool to detect faint moving objects in CCD-frames.

The CoLiTex group developed also a new tool for the calibration of astronomical images FrameSmooth as a cross-platform module for brightness equalization of CoLiTec software. It allows processing images with any formats. Module is based on the filter for brightness equalization, inverse median, and nonlinear high-frequency filters. It supports additional astronomical master-frames (Bias, Dark, DarkFlat and Flat) as well as the OLDAS mode and tools for converting images in fits format were added (http://www.neoastrosoft.com/filter_en/). Advantages of this method as comparing with traditional Muniwin calibration method are illustrated in Fig. 1.

Special-oriented software has been developed for variable star research at the Odesa National Maritime University by I. Andronov and his collaborators. 


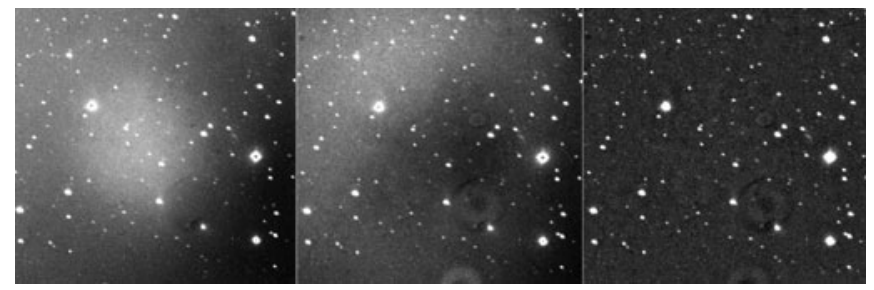

Figure 1. Field of variable star V1323 Her. From left to right: raw image, calibrated in usual way with the Muniwin, calibrated with a new FrameSmooth software

The program MCV ("Multi-Column View") allows to make time series analysis of irregularly spaced data, particularly, having unique features: the approximation using combinations of algebraic and trigonometric polynomials simultaneously for up to three main periods (and harmonics); the periodogram analysis using the trigonometric polynomial of an user-defined degree superimposed on to an algebraic polynomial trend without a pre-whitening; the reduction of the CCD-measurements using many stars in the field (the algorithm of the "artificial comparison star") and dozens of other features. At minimum, the program may be used for an automatic visualization of the multi- or one-column time series and preparation of graphs in the raster format. The description of the first version was published by Andronov \& Baklanov 2004, but the program is regularly upgraded and new versions are available (http://soft. softodrom.ru/ap/Bred2i-p4726).

Breus 2007 has proposed the VSCalc ("Variable Stars Calculator") software, where 1) reduction of visual observations and conversion of the photographic plate numbers in HJD are provided (currently the database of the Odesa astroplate collection is available, but other files may be used as well); 2) the periodogram analysis may be conducted with the method by Lafler-Kinman-Kholopov; 3) the extrema may be determined using an algebraic polynomial of statistically optimal order. This method was improved for the multi-color observations as well as additional algorithms were realized for vicinities of the extrema and for complete light curves by Andronov et al. 2016. New programs are developed also for automatic search for variable stars using measurements of all stars in the field and for the photometry of stars using overlapping images. These programs may be revised upon request.

\section{UkrVO and VIRGO Astronomical Web-services}

From the analysis of such a large number of web interfaces it can be noted that the main progress in their development is associated with easier access to data and improving their visualization. Besides these two aspects, it was introduced a new approach to the quality of data provided by the user. In our opinion, one of the main reasons that an obstacle to the use of big data in scientific studies, there is uncertainty value bias, which is usually not considered by the available standardized software.

For example, despite the overall success of the VIRGO web-service project, a number of the Ukrainian astronomers, who use the data of X-ray and gamma-ray observatories, is still small. To overcome this problem, a web-based virtual observatory VIRGO as a "sky map" (http://skyview.virgoua.org) was developed by V. Savchenko and D. Savchenko (Fig. 2) . It allows visualizing the distribution parameter (intensity of signal, etc.) in space and the range energies setting by the user. One of the examples of using these sky maps in 2-5 and 5-10 keV range with a public database of X-ray observations by XMM-Newton is a search of the dark matter decay line (Boyarsky et al. 2014, Savchenko \& Iakubovskyi 2014). This web-based observatory contains also various catalogues of X-ray extragalaxy sources (active galaxy nuclei, galaxy clusters) compiled at the Main 


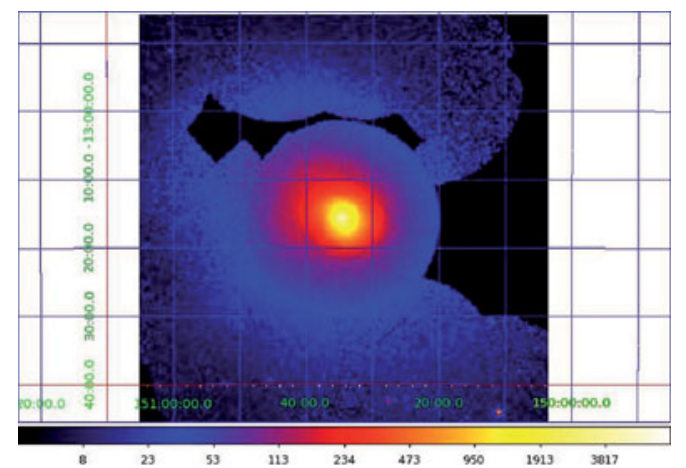

Figure 2. Example of the sky map from VIRGO web-site: a $2-5 \mathrm{keV}$ image of 1 square degree around Perseus galaxy cluster (Savchenko \& Iakubovskyi (2014)).

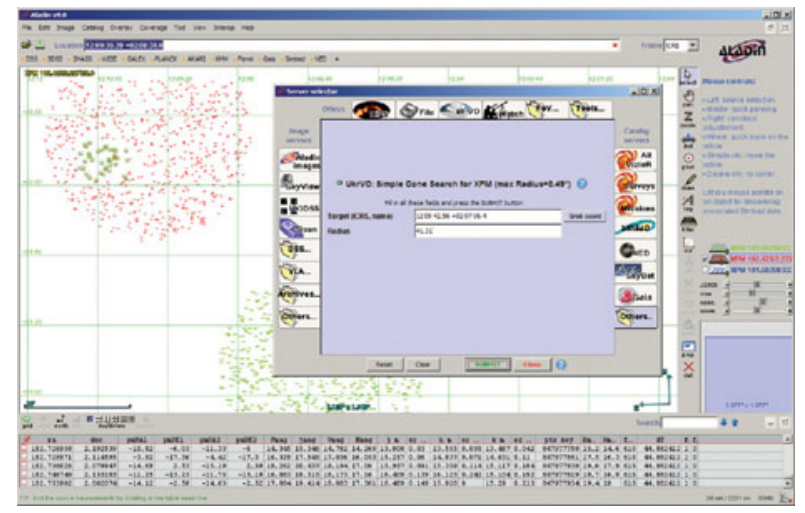

Figure 3. Screenshot of the GUI and search results for XPM catalogue obtained with Aladin (AWS is developed by Mazhaev \& Protsyuk (2013)).

Astronomical Observatory of the National Academy of Sciences of Ukraine (MAO NASU) (see, for example, Chesnok et al. 2009, Babyk et al. 2014, Babyk \& Vavilova 2014), Pulatova et al. 2015, Vasylenko et al. 2015).

The VizieR web service (Strasbourg, France) contains more than 15400 published astronomical catalogues. The Astronomical Web-Services (AWS) of UkrVO prepared by Mazhaev \& Protsyuk 2013 provide the automated search and selection of required data in accordance with the SCS standard for the catalogues compiled in Ukraine. We refer to the All-Sky Compiled Catalogue of 2.5 million stars, ASCC (Kharchenko N.V., Roeser S.), Astrographic Catalogue of 19451751 stars and galaxies, FONAC (Andruk et al. 2016), observed in frame of the FON project (photogtaphic sky survey), and Catalogue of the absolute proper motions of about 280 million stars, XPM (Fedorov et al. 2009). The structure of AWS web address consists of path to script and search options after the question mark. The user can search in different areas of the sky and retrieve the data from the astrometric catalogues. These AWS were inscribed into a register of the USA Virtual Astronomical Observatory (VAO). Using Data Discovery Tool (DDT) developed by the VAO, it is possible to retrieve data from all archives, which are inscribed in the VAO register. Illustration of the access with AWS by using Graphical User Interface (GUI) of Aladin to the XPM catalogue is given in Fig. 3. It's also possible with this GUI to obtain access to two UkrVO databases of observations with astro photo plates and CCDs conducted in the 20th-21st centuries, respectively (see, text below, in detail). 


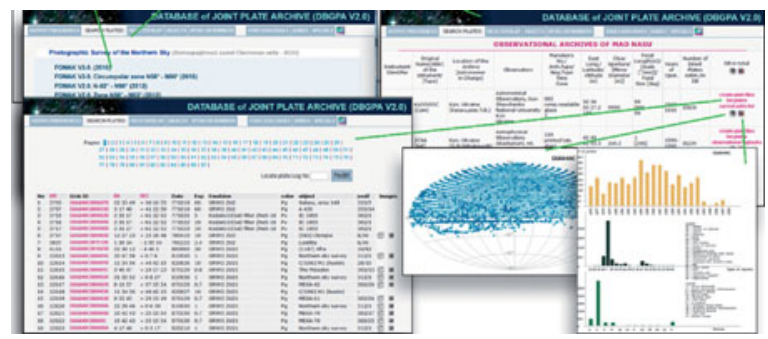

Figure 4. Screenshot of the interface of the UkrVO Joint Digitized Archive of astroplates, $\mathrm{N}=46607$ up to the current date (http://gua.db.ukr-vo.org/archivespecial.php).

The UkrVO's development allows us 1) to save the unique astronomical observational heritage accumulated in observatories of Ukraine from the 1890-ies; 2) to open the wide on-line access to the joint database of digitized astronomic negatives and spectra for the national/foreign scientific community. The current state is available at the UkrVO web-site. At the initial stage, this task has concluded in the creation and development of the UkrVO Joint Digital Archive (UkrVO JDA) of photographic observations. The total number of photographic plates in the UkrVO joint collection exceeds 300 thousands, including not only the positional but also the spectral and photometric observations. The current JDA version includes, at mainly, the positional observations of various celestial bodies. About 125,000 astroplates (Ukrainian Plate Archives) are included in the WideField Plate Database (http://www.wfpdb.org/catalogue.html). Now the UkrVO JDA gives access to about of 50,000 digitized astroplates of a good quality (its interface is illustrated in Fig. 4. The UkrVO JDA Data releases, which were performed by scientists from Kyiv, Mykolaiv, Odesa, L'viv, and Crimea including those in a tight international cooperation, are in a final stage for issuing. UkrVO Data Release 1 deals with the star objects (FONAC, Polar zone, open clusters, star fields in vicinity of gamma-ray bursts, etc.). UkrVO Data Release 2 deals with the Solar System bodies (giant and small planets, satellites of giant planets, astronomical heritage images, etc.). Source of these data: the digitized astroplates, which were organized as the UkrVO JDA database of astroplate network collections of different institutions as owners of these collections.

The data archives and catalogues conducted at the Crimean Astrophysical Observatory and formalized as the Crimean Astrophysical Virtual Observatory (CrAVO) are the part of the UkrVO astroinformatics resource. The CrAVO database consists of 39 catalogues: some of them are digitized, 16 catalogues with the data on magnitudes and spectral classes of 34154 stars ("Shajn Plan") are in processing Slyapnikov et al. 2015. Information about these archives of astronegatives is placed also at the International Center in Sofia (http://www.skyarchive.org/); the data are available through a database in the VizieR catalog VI/ 90 or with CRI key at: http://draco.skyarchive.org/ search/.

For many years the MAO NASU has been involved into services for determinitaion of the Earth Orientation Parameters (EOP), namely, polar motion coordinates and UT1 variations. Observatory takes part in the International Earth Rotation and Reference System Service (IERS), in particular, for creating the EOP IERS C01 database (Yatskiv 1985). It is presented as a series of these parameters given at 0.1 year intervals from 1846 to 1889 (polar motion only) and 0.05 year interval from 1890 until now (polar motion, celestial pole offsets, UT1-UTC since 1962). For many decades, the observations were made using mostly visual and photographic zenith telescopes. Since the advent of the space era in the 1960's, new geodetic techniques were applied for geodynamics. Now, the global observing activity involves VLBI, Lunar (LLR) and 
Satellite Laser Ranging (SLR), Global Positioning System (GPS) and, more recently, Doppler Orbitography and Radiopositioning Integrated by Satellite (DORIS). So, the C01 series is a composite series based on the temporal solutions by Fedorov et al. 1972 for the Earth polar motion and derived from three series of absolute declination programs in Pulkovo, Greenwich, Washington (Bolotin \& Yatskiv 2003). The current contribution of the MAO NASU to the IERS database is as follows: 1) MAO SLR Database (started on Feb 1, 2013), two SLR stations, raw data (frd); database is supervised by M. Medvedsky (http://eop.mao.kiev.ua/robots/basa.php); 2) MAO GNSS database (started on Dec 1, 1997), 18 permanent stations, 1s and 30s observational intervals; database is supervised by O. Khoda (ftp://ftp.mao.kiev.ua/pub/gnss).

Acknowledgements. This work was partially supported in frame of the Target Complex Program of Scientific Space Research of the National Academy of Sciences of Ukraine (2012-2017). The first two authors of this paper thank the IAU and the OrgCommittee of the IAU Symp. 325 for the financial support.

\section{References}

Andronov, I. L. \& Baklanov, A. V. 2004, Astronomical School's Report, 5, 264

Andronov, I. L., et al. 2016, Phys. Journal, 2, 140.

Andruk, V. M., et al. 2016, Kinemat. Phys. Celestial Bodies, 32, 261

Babyk, Yu. V., et al. 2014, Astronomy Reports, 58, 587.

Babyk, Yu. V. \& Vavilova, I. B. 2014, Astrophys. \& Space, Sci., 349, 415.

Bolotin, S. \& Yatskiv, Ya. 2003, IVS Annual report-NASA-TP-2003-211619, Greenbelt, p. 269272

Boyarsky, A., et al. 2014, Phys. Rev. Lett., 113, 251301

Breus, V. V. 2007, Odessa Astron. Publications, 20, 32

Borne, K., et al. 2009, The Astronomy and Astrophys. Decadal Survey, Position Papers, no. 6

Brescia, M. \& Longo, G. 2013, Nuclear Instruments and Methods in Physics Research A, 720, 92

Butler, D. 2007, Nature, 446, 354

Cavuoti, S., et al. 2012, Proceedings of the SPIE, 8451, article id. 845103

Chesnok, N. G., et al. 2009, Kinemat. Phys. Celest. Bodies, 25, 107

Eastman, T., et al. 2005, Data Science Journal, 4, 67

Fedorov, E. P., et al. 1972, Motion of the earth's pole from 1890.0 to 1969.0, Kiev, Nauk. dumka

Fedorov, P., Myznikov, A., \& Akhmetov, V. 2009, MNRAS, 393, 133

Feigelson, E. D., et al. 2013, In: ADASS XXII, ASP Conferences Series, 15

Goodman, A., et al. 2012, In: ADASS XXI, ASP Conference Series, 461, 267

Gray, J., et al. 2005, arxiv.org/abs/cs/0502008

Kharchenko, N. V. \& Roeser S. 2001, Kinemat. Phys. Celestial Bodies, 17, 409

Mazhaev, A. E. \& Protsyuk, Yu. I. 2013, Odessa Astron. Publications, 26/2, 233

Michaelian, A. 2016, Baltic Astronomy, 25(1), 75

Norris, R. P., et al. 2013, PASA, 30, 20

Pepe, A., et al. 2014, PLoS ONE 9(8): e104798. doi:10.1371/journal.pone.0104798

Pulatova, N. G., et al. 2015, MNRAS, 447, 2209

Ruiz, J. E., et al. 2014, Astronomy and Computing, 7, 3

Savanevych, V. E., et al. 2015a, MNRAS, 451, 3, 3287

Savanevych, V. E., et al. 2015b, Kinemat. Phys. Cel. Bodies, 31(6), 302

Savchenko, D. \& Iakubovskyi, D. 2014, AASP, 4, 51

Slyapnikov, A. A., et al. 2015, Baltic Astron., 24, 462

Vasylenko, A. A., et al. 2015, Astrophys. ES Space Sci., 360, article id.37

Vavilova, I. B., et al. 2012a, Kinemat. Phys. Cel. Bodies, 28(2), 85

Vavilova, I. B., et al. 2012b, Baltic Astronomy, 21/3, 356

Vavilova, I. B. 2016, Odessa Astron. Publications, 29, 109

Yatskiv, Ya.S. 1985, Report of IAU Com. 19: Rotation of the Earth, Trans. IAU, 19A, 193 